\title{
How Does Unintentional Eye Contact with a Robot Affect Users' Emotional Attachment to It?: Investigation on the Effects of Eye Contact and Joint Attention on Users' Emotional Attachment to a Robot
}

\author{
Takanori Komatsu ${ }^{1}$ and Haruka Takahashi ${ }^{2}$ \\ ${ }^{1}$ Department of Frontier Media Science, Meiji University \\ Nakano 4-21-1, 164-8525 Tokyo, Japan \\ ${ }^{2}$ Graduate School of Science and Technology, Shinshu University \\ Tokida 3-15-1, 386-8567 Ueda, Japan \\ tkomateacm.org
}

\begin{abstract}
Eye contact behavior plays a significant role in establishing intimate interaction between a user and a robot. In this study, more specifically, we assumed that unintentional eye contact with a robot would make a person feel a stronger emotional attachment to the robot, especially when the user believes that $\mathrm{s}$ /he had achieved joint attention with the robot. To verify this assumption, we developed an experimental setting to make users establish joint attention and eye-contact with a robot. We then conducted an experiment to investigate the above assumption; that is, independent variables were with/without eye-contact and with/without joint attention, while the dependent variable was a questionnaire that consisted of a love-liking scale. The results showed that our assumption was verified.
\end{abstract}

Keywords: eye contact, joint attention, emotional attachment, human-robot interaction.

\section{Introduction}

In our daily lives, we feel emotional attachment to someone with whom we make unintentional eye contact. Imagine that you and your friend are relaxing at a streetfacing cafe. A smartly dressed executive-type person then walks past this cafe, but unfortunately, his fly is fully open, and both of you notice this. Then, you glance at your friend and vice versa, so this means that you and your friend establish eye contact with each other unintentionally. Eventually, both of you burst into laughter. In this case, you will feel some emotional attachment to your friend and vice versa, and you might exchange high fives saying "hey man, did you see? What a stupid dude!"

In terms of the above example, we assume that eye contact and joint attention play a significant role. Eye contact is when one meets another's eyes and vice versa $[1,2]$. 
Joint attention is achieved by sharing visual attention with another towards the same object or event. Moreover, the most important thing about joint attention is not only that the both of you are looking at the same object or event but also that you each know that the other is attending to the same object $[3,4,5]$. It is thought that both eye contact and joint attention have a strong influence on social behaviors, cognitive development, and language acquisition [6,7].

In this study, we assumed that unintentional eye contact between individuals who have already achieved joint attention facilitates their intimate relationship. Let us explain this assumption by using the above example. Here, it can be said that you and your friend looked at the same object or event (e.g., fully opened fly). If you do not know that your friend is also looking at the same object, nothing would happen after the unintentional eye contact between you and your friend because both of you did not establish joint attention. On the other hand, if you know that your friend is looking at the same object and vice versa, you and your friend might burst into laughter immediately after unintentional eye contact because you already succeeded in establishing joint attention with your friend. Thus, it is reasonable to assume that unintentional eye contact after establishing joint attention would play a significant role in facilitating their interactions.

Furthermore, we believe that this assumption can be applied to the domain of human-robot interaction. Up to now, several studies in human-robot interaction have tried to implement specific functions to establish eye contact with humans in order to realize intimate interaction $[8,9]$. Most of these studies showed face to face interaction between humans and robots, and there are no studies that focus on our assumption; that is, to observe how unintentional eye contact and joint attention affect users' impressions of robots.

The purpose of this study is then to investigate the effects of unintentional eye contact and joint attention on users' emotional attachment to robots. Specifically, we conducted an experiment to observe how 2 (with/without unintentional eye contact) $\times$ 2 (with/without joint attention) factors affected the participants' emotional attachment to a robot. The results of this experiment should contribute to a strategy for establishing intimate interaction between humans and robots.

\section{Experiment}

\subsection{Procedure}

We used a treasure hunting video game environment as an experimental setting to investigate the participants' emotional attachment to a robot. In this game, a game image scrolls forward on a straight road with three treasure boxes appearing along the way (Figure 1). The participants were told that a gold coin is inside one of three treasure boxes, while the other two contain nothing, and that the game ends after the participants encounter 40 trials (actually, the game ends after 28 trials. See later description.). The purpose of this game is to get as many coins as possible. 

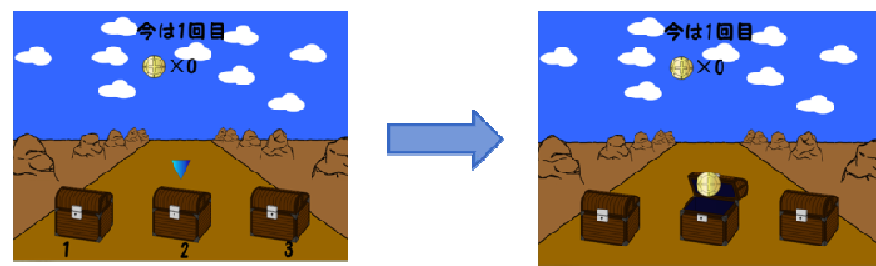

Fig. 1. Treasure hunting video game. (left) A participant selects the 2nd box, and (right) there was a gold coin in this box.

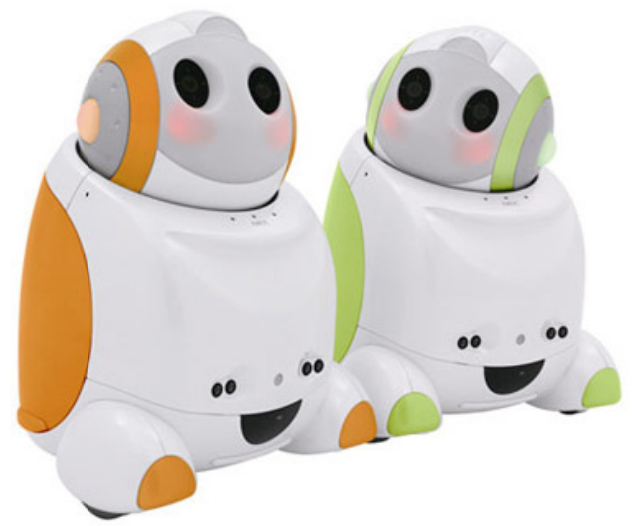

Fig. 2. PaPeRo robot (NEC Corporation)

In each trial, a robot (PaPeRo robot, NEC Corporation, Figure 2) placed left next to the participant told them which box it expects the gold coin to be in by using speech sounds. The game was projected not on a normal computer display but on a Tobii T60 Eye Tracker (Tobii Technology AB) that can capture where participants are gazing on the computer display when playing the treasure hunting video game. Therefore, the robot's suggestions were based on the treasure box the participants were actually looking at. The participants were told that this robot is a collaborator and they could freely accept or reject the robot's suggestions. After the participants selected one box among the three by using a computer mouse, they could immediately know whether the selected box contained a coin or not on the display.

During the game, we prepared two unexpected events: one is that the selected treasure box contains tens of gold coins in a randomly selected trial, and another is that the selected box contains skeleton bones in the 28th trial and the game is suddenly finished. In contrast with the example story mentioned in the Introduction, the participant is "you," the robot is "your friend," and the unexpected event is "fully opened fly."

The actual purpose of this experiment was to comprehend the participants' emotional attachment to the robot, especially whether eye contact and joint attention affected this emotional attachment. Therefore, after playing the game, the participants were asked to 
fill in a questionnaire on their impressions of the robot. The questionnaire consisted of eight questions using a 9-point Likert scale (maximum evaluation: 9 points; minimum evaluation: 1 point; see Table 1). Here, Q1 "I established eye contact with this robot" and Q2 "This robot is watching the same target as me" were used as manipulation checks to see whether eye contact and joint attention were properly achieved, respectively. The total score of the remaining six questions (Q3 - Q8) were used as the participants' "emotional attachment score" of this robot; that is, more points meant a better impression of the robot (the highest score was 54 points, and the lowest was 6 points). Actually, these six questions consisted of a modified love-like scale [10].

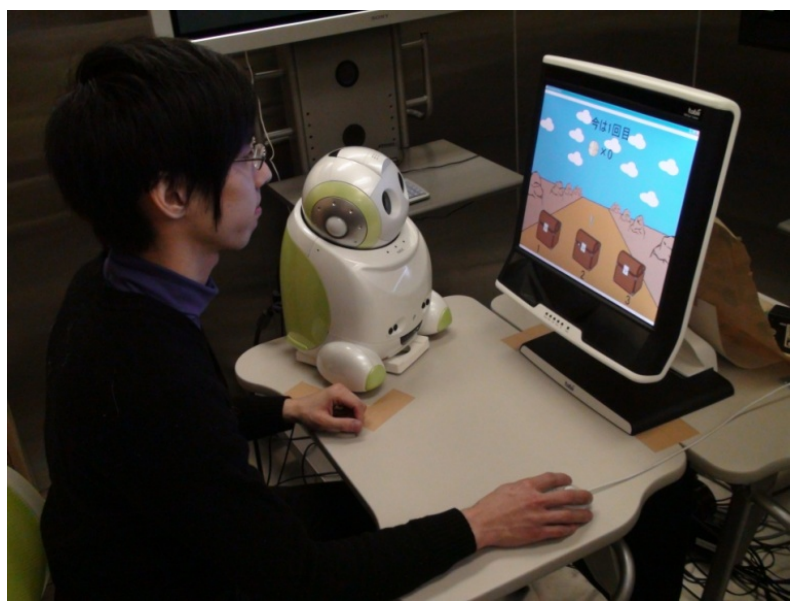

Fig. 3. Experimental scene

Table 1. Questionnaire Used

Q1: I established eye contact with this robot.

Q2: This robot is watching the same target as me.

Q3: My feeling is the same as usual even if I use this robot.

Q4: This robot has good adaptability.

Q5: This robot deserves to work on responsible tasks.

Q6: This robot is always preferred among similar robots.

Q7: This robot makes favorable impressions on many people.

Q8: I prefer this robot because its thinking is similar to my way of thinking 

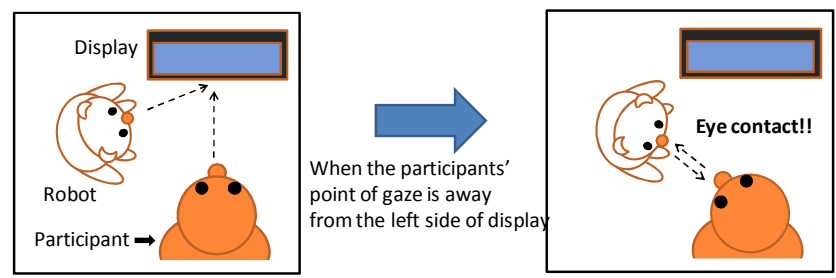

Fig. 4. Eye contact functions in the experimental setting

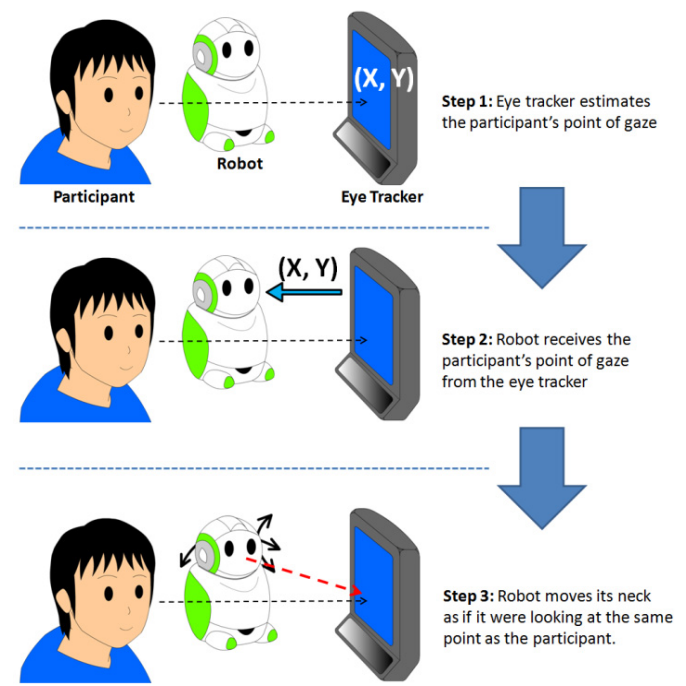

Fig. 5. Joint attention functions in this experimental setting

\subsection{Eye Contact and Joint Attention Functions in the Robot}

In this experiment, we designed the robot's functions to realize eye contact and joint attention with the participants. First, to realize the robot's eye contact functions with the participants, we designed the robot to turn to the participants when the participants' point of gaze was away from the left side of display. Since the robot was placed to the left next to the participants, participants whose gaze went to the left of the display established eye contact with this robot (Figure 4). We expected that the participants would unintentionally glance at the robot when the unexpected event happened during the game. In this case, the participants would turn to the left to glance at the robot, so their point of gaze would be away from the left side of the display. Then, if the robot turned to the participants simultaneously, they would establish unintentional eye contact when the unexpected event happened.

Then, to realize joint attention between them, we designed robot to function that its head moves as if the robot were following the participants' point of gaze during the game. Actually, the robot continuously received the actual coordinates of the participants' point of gaze from the eye tracker, and the angle of the head joints 
corresponded to these received coordinates as if the robot were looking at the same point as the participant (Figure 5). We expected that such functions would make the participants believe like "this robot is looking at the same treasure box during this game," and this would also make the participants believe that they had actually established joint attention.

\subsection{Participants}

Forty Japanese university students participated (30 men and 10 women; 19-24 years old). These participants were randomly divided into the following four experimental groups.

- Group 1 (10 participants): These participants played the treasure hunting video game with the robot that had both eye contact and joint attention functions. This means that the participants interacted with the robot, which behaved as if it were looking at the same treasure box as the participants, and that it glanced at the participants when the unexpected event happened.

- Group 2 (10 participants): These participants played the game with the robot that had only the joint attention function. This means that the participants interacted with the robot, which behaved as if it were looking at the same box but did not react to the unexpected event.

- Group 3 (10 participants): These participants played the game with the robot that had only the eye contact function. This means that they interacted with the robot, which glanced at them but did not behave as if it were looking at the same box.

- Group 4 (10 participants): These participants played the game with the robot that had neither the eye contact or joint attention functions.

During this experiment, the robot suggested to the participants which box it expected the gold coin to be in regardless of whether it had the joint attention function, so there were no differences in terms of the robot's suggestions among the experimental conditions.

This experiment was a 2 (eye contact factor: with/without eye contact functions) $\times$ 2 (joint attention factor: with/without joint attention functions) between-subject design. Independent variables were the above two factors (eye contact and joint attention factors), while a dependent variable was the emotional attachment scores (the total score from Q3 to Q8 in Table 1).

\subsection{Manipulation Check}

First, we checked whether the experimental conditions were set properly. Specifically, this required confirming whether the participants believed that they had established eye contact with the robot when the unexpected events happened (Groups 1 and 3) or whether they believed that they had succeeded in establishing joint attention between the robot and themselves (Groups 1 and 2). 
The scores for Q1, "I established eye contact with this robot," in Table 1 are shown in Figure 6. Specifically, the average score for Group 1 was $7.4(\mathrm{SD}=1.2)$, that of Group 2 was $3.2(\mathrm{SD}=2.2$ ), Group 3 was 6.6 ( $\mathrm{SD}=1.6)$, and Group 4 was 3.7 $(\mathrm{SD}=2.1)$. The scores for $\mathrm{Q} 1$ were then analyzed by using a one-way ANOVA (between subject plans, independent variable: four experimental groups (Groups 1 to 4), dependent variable: score of Q1). The results of the ANOVA showed significant differences among the experimental groups $[\mathrm{F}(3,39)=12.00, \mathrm{p}<.01(* *)]$, and a multiple comparison test by using an LSD test showed that the scores in Groups 1 and 3 were significantly higher than those in Groups 2 and 4 [MSe $=3.6250, \mathrm{p}<.05(*)]$. Therefore, we clearly observed that the participants in Groups 1 and 3, in which the eye contact functions were implemented in the robot, showed they believed that they had established eye contact with the robot.

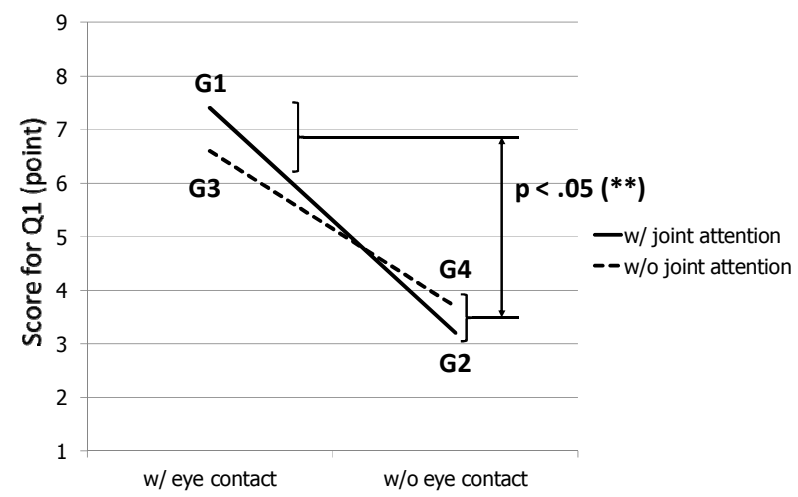

Fig. 6. Scores for Q1 "I established eye contact with this robot" for each experimental group

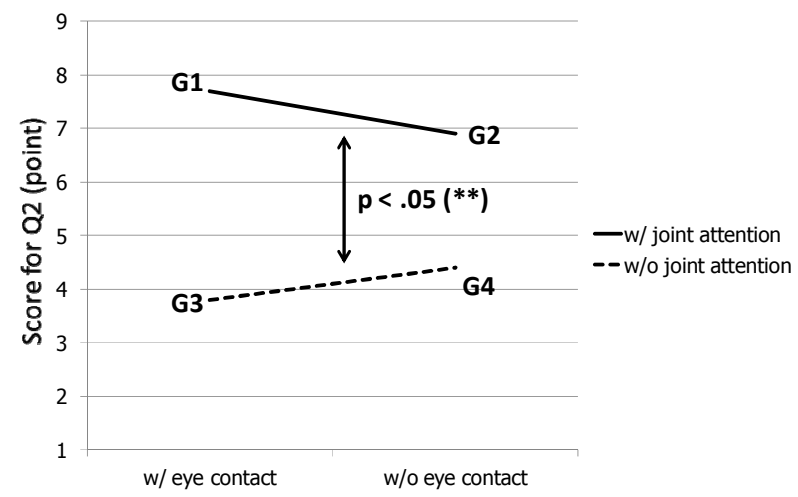

Fig. 7. Scores for Q2 "This robot is looking at the same target as me" for each experimental group

The scores for Q2, "This robot is looking at the same target as me," are shown in Figure 7. Specifically, the average score for Group 1 was 7.7 (SD = 1.6), that of Group 2 was $6.9(\mathrm{SD}=2.0)$, Group 3 was $3.8(\mathrm{SD}=1.7)$, and Group 4 was $4.4(\mathrm{SD}=2.2)$. 
The scores for Q2 were then analyzed by using a one-way ANOVA (between subject plans, independent variable: four experimental groups (Groups 1 to 4), dependent variable: score for Q2). The results of the ANOVA showed significant differences among the experimental groups $[\mathrm{F}(3,39)=9.27, \mathrm{p}<.01(* *)]$, and a multiple comparison test by using an LSD test showed that the scores in Groups 1 and 2 were significantly higher than those in Groups 3 and 4 [MSe $=3.8611, \mathrm{p}<.05(*)]$. Therefore, we clearly observed that the participants in Groups 1 and 2, in which the joint attention functions were implemented in the robot, showed that they believed that they had established joint attention between them.

Therefore, we could confirm that the experimental conditions were properly set; that is, the participants believed that they had established eye contact with the robot when the unexpected events happened (Groups 1 and 3), and they believed that they had established joint attention between the robot and themselves (Groups 1 and 2). Thus, we could focus purely on the effects of eye contact and joint attention on the participants' emotional attachment to the robot.

\section{$2.5 \quad$ Results}

The participants' emotional attachment scores are shown in Figure 8. Specifically, the average score for Group 1 was 32.0 ( $\mathrm{SD}=3.6$ ), that of Group 2 was 29.6 ( $\mathrm{SD}=5.6$ ), Group 3 was 26.8 ( $\mathrm{SD}=6.2$ ), and Group 4 was 30.9 ( $\mathrm{SD}=5.4)$. These scores were then analyzed by using a $2 \times 2$ ANOVA (between subject plans, independent variable $\# 1$ : eye contact factor, independent variable \#2: joint attention factor, dependent variable: emotional attachment scores). The results of the ANOVA showed marginal difference in the interaction effect $[\mathrm{F}(1,39)=3.44, \mathrm{p}<.10(+)]$ but no significant difference in the main effect of the eye contact factor $[\mathrm{F}(1,39)=0.23$, n.s. $]$ and of joint attention factor $[F(1,39)=1.24$, n.s.]. The simple main effects of the two independent variables were then analyzed, and the results showed significant differences in the scores between Groups 1 and $3[\mathrm{~F}(1,39)=4.40, \mathrm{p}<.05(*)]$; that is, the robot that had only the eye contact function showed lower emotional attachment scores.

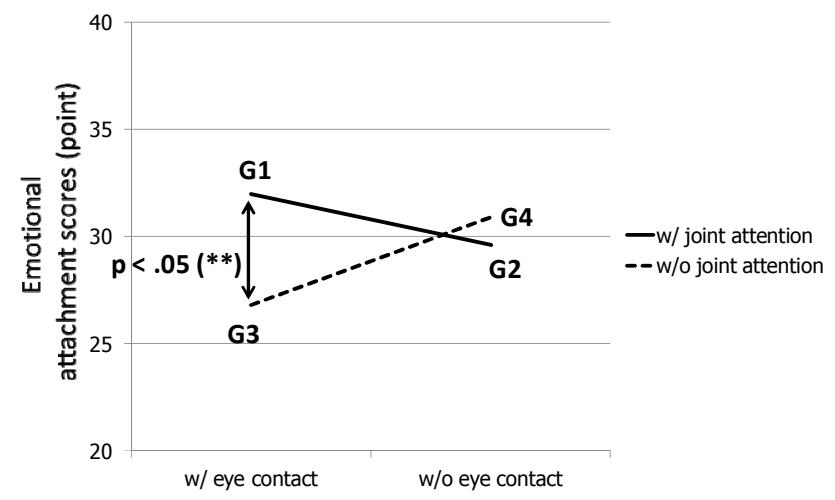

Fig. 8. Participants' emotional attachment scores for each experimental group 
As the results of the experiment, we could confirm the following.

- Eye contact and joint attention functions did not greatly affect the participants' emotional attachment scores.

- The participants who interacted with the robot that had both eye contact and joint attention functions (Group 1) showed rather higher scores compared with the other groups, but there was no significant differences with those who interacted with the robot that had neither of the functions (Group 4).

- However, the participants who interacted with the robot that had only the eye contact function (Group 3) showed significantly lower scores compared with those with both functions.

\section{Conclusions}

Eye contact behavior plays a significant role in establishing intimate interaction between a user and a robot. In this study, more specifically, we assumed that unintentional eye contact with the robot would make a person feel stronger emotional attachment to the robot, especially when the user believed that they had achieved joint attention. To verify this assumption, we developed an experimental setting to make users establish joint attention and eye contact with the robot. We then conducted an experiment to investigate the above assumption; that is, independent variables were with/without eye contact and with/without joint attention, while the dependent variable was a questionnaire consisting of a love-liking scale.

As the results of the experiment, we could confirm that the eye contact and joint attention functions did not greatly affect the participants' emotional attachment scores. Moreover, the participants who interacted with the robot that had both eye contact and joint attention functions showed rather higher scores compared with the other groups, but there were no significant differences with those who interacted with the robot that had neither of the functions. However, the participants who interacted with the robot that had only the eye contact function showed significantly lower scores compared with the ones with both functions.

The results of this study then succeeded in showing that eye contact and joint attention functions are significant cues for users' emotional attachment to a robot; however, only the eye contact function without joint attention did was not.

Acknowledgments. This study was partially supported by the Special Coordination Funds for Promoting Science and Technology from the Ministry of Education, Culture, Sports, Science and Technology (MEXT), Japan.

\section{References}

1. Argyle, M., Dean, J.: Eye-Contact, Distance and Affiliation. Sociometry 28(3), 289-304 (1965)

2. Kleinke, C.L.: Gaze and eye contact: A research review. Psychological Bulletin 100(1), 78-100 (1986) 
3. Scaife, M., Bruner, J.: The capacity for joint visual attention in the infant. Nature 253, 265-266 (1975)

4. Tomasello, M.: Joint attention as social cognition. In: Moore, C., Dunham, P.J. (eds.) Joint Attention, Its Origins and Role in Development, Lawrence Erlbaum, Hillsdale (1995)

5. Argyle, M., Ingham, R.: Mutual gaze and proximity. Semiotica 6, 32-49 (1972)

6. Tomasello, M., Farrar, M.J.: Joint Attention and Early Language. Child Development 57(6), 1454-1463 (1986)

7. Moralesa, M., Mundyb, P., Delgadob, C.E.F., Yaleb, M., Messingerb, D., Nealb, R., Schwartzb, H.K.: Responding to Joint Attention Across the 6- Through 24-Month Age Period and Early Language Acquisition. Journal of Applied Developmental Psychology 21(3), 283-298 (2000)

8. Kozima, H.: Infanoid: A babybot that explores the social environment. In: Dautenhahn, K., Bond, A.H., Canamero, L., Edmonds, B. (eds.) Socially Intelligent Agents: Creating Relationships with Computers and Robots, pp. 157-164. Kluwer Academic Publishers, Amsterdam (2002)

9. Yonezawa, T., Yamazoe, H., Utsumi, A., Abe, S.: Evaluating Crossmodal Awareness of Daily-partner Robot to User's Behaviors with Gaze and Utterance Detection. In: Proc. of the 3rd ACM International Workshop on Context-Awareness for Self-Managing System (CASEMANS 2009), pp. 1-8 (2009)

10. Rubin, Z.: Measurement of romantic love. Journal of Personality and Social Psychology 16(2), 265-273 (1970) 